\title{
Comparison of hyperemic efficacy between femoral and antecubital fossa vein adenosine infusion for fractional flow reserve assessment
}

\author{
Jacek Legutko, Paweł Kleczyński, Artur Dziewierz, Lukasz Rzeszutko, Dariusz Dudek \\ Institute of Cardiology, Jagiellonian University, Krakow, Poland
}

Adv Interv Cardiol 2019; 15, 1 (55): 52-58

DOI: https://doi.org/10.5114/aic.2019.83652

\begin{abstract}
A bstract
Introduction: Intravenous infusion of adenosine via the femoral vein is commonly used to achieve maximum hyperemia for fractional flow reserve (FFR) assessment in the catheterization laboratory. In the era of transradial access for coronary interventions, obtaining additional venous access with sheath insertion in the groin is unpractical and may be associated with a higher risk of bleeding complications. In a vast majority of cases, patients scheduled for the catheterization laboratory are already equipped with peripheral vein access in antecubital fossa vein. However, only limited data exist to support non-central vein infusion of adenosine instead of the femoral vein for FFR assessment.

Aim: To compare infusion of adenosine via a central versus a peripheral vein for the assessment of peak FFR.

Material and methods: We enrolled 50 consecutive patients with 125 borderline coronary lesions that were assessed by FFR using adenosine femoral and antecubital vein infusion of $140 \mu \mathrm{g} / \mathrm{kg} / \mathrm{min}$.

Results: Physiological severity assessed with femoral vein adenosine infusion at $140 \mu \mathrm{g} / \mathrm{kg} / \mathrm{min}$ was mean $0.82 \pm 0.09$, and with antecubital vein adenosine infusion at $140 \mu \mathrm{g} / \mathrm{kg} / \mathrm{min}$ was $0.82 \pm 0.09$. The mean time from initiation of adenosine infusion to maximal stable hyperemia was significantly shorter for $140 \mu \mathrm{g} / \mathrm{kg} / \mathrm{min}$ femoral vein infusion as compared to antecubital vein infusion ( $49 \pm 19$ s vs. $68 \pm 23$ s; $p<0.001$ ). There was a strong correlation between FFR values obtained from $140 \mu \mathrm{g} / \mathrm{kg} / \mathrm{min}$ femoral and antecubital vein infusion $(r=0.99 ; p<0.001)$.

Conclusions: Antecubital vein adenosine infusion achieved FFR values are very similar to those obtained using femoral vein adenosine administration. However, time to maximal hyperemia is longer with infusion via the antecubital vein.

Key words: coronary artery disease, adenosine, fractional flow reserve, borderline lesion, physiology.

Sum mary

Intravenous infusion of adenosine via the femoral vein is commonly used to achieve maximum hyperemia for fractional flow reserve (FFR) assessment in the catheterization laboratory. In the era of transradial access for coronary interventions, obtaining additional venous access for sheath insertion in the groin is impractical and may be associated with a higher risk of bleeding complications. In a vast majority of cases, patients scheduled for the catheterization laboratory are already equipped with peripheral vein access in the antecubital fossa vein. However, only limited data exist to support non-central vein infusion of adenosine instead of femoral vein infusion for an all-comers population of patients and lesions. The purpose of this study was to compare infusion of adenosine via a central versus a peripheral vein for the assessment of peak FFR.
\end{abstract}

\section{Introduction}

Coronary pressure-derived fractional flow reserve (FFR) is the current standard of care for the functional assessment of lesion severity in patients with intermediate-grade stenosis (typically around $40-90 \%$ stenosis) without evidence of ischemia in non-invasive testing, or in those with multivessel disease [1-6]. The FFR value of $\leq 0.80$ defines hemodynamically relevant lesions requiring revascularization, while the FFR threshold of $\leq 0.75$ is used to define more severe ischemia that is of prognostic relevance. Achievement of maximal hyperemia is crucial for appropriate and accurate FFR assessment and can be

\section{Corresponding author:}

Pawel Kleczynski MD, PhD, Institute of Cardiology, Jagiellonian University, 17 Kopernika St, 31-501 Krakow, Poland, phone: +48 124247181 , fax: +48124247184 , e-mail: kleczu@interia.pl

Received: 3.02.2019, accepted: 15.02.2019. 
obtained either with intravenous infusion of adenosine via a central vein (usually femoral) or intracoronary boluses of adenosine [7]. In the era of transradial access for percutaneous coronary interventions $(\mathrm{PCl})$, obtaining additional venous access for sheath insertion in the groin is impractical and may be associated with a higher risk of complications if the patient has already received heparin. In a vast majority of cases, patients scheduled for the catheterization laboratory are already equipped with peripheral vein access in the antecubital fossa vein, which can also be used for adenosine infusion. However, only limited data exist to support non-central vein infusion of adenosine instead of femoral vein infusion for an all-comers population of patients and lesions $[8,9]$.

\section{Aim}

The purpose of this study was to compare infusion of adenosine via a central vs. a peripheral vein for the assessment of peak FFR.

\section{Material and methods}

Consecutive patients with stable angina between 18 and 90 years of age with angiographically borderline stenosis (40-90\% diameter stenosis by visual assessment) in a major epicardial coronary artery, who were scheduled for FFR, were prospectively enrolled. Baseline clinical data were collected. Patients with acute myocardial infarction in the prior 30 days or contraindication to adenosine were excluded. Ethics approval was granted from the institutional ethics review process and all patients gave written informed consent. Coronary angiography was performed with the femoral or radial approach based individual operator preferences. All procedures were performed by experienced operators in a cardiac reference university center with > 100 FFR assessments per year each. Patients had intravenous access established before the pressure-wire procedure using a pink (20-gauge) cannula placed in the antecubital fossa vein. For the femoral venous administration of adenosine, a $6 \mathrm{Fr}$ venous sheath with a side arm was used. The infusion system was filled with adenosine to exclude the wash-out period of the saline. The s5/s5i console and Verrata Pressure Guide Wire (Philips Volcano Corporation, San Diego, California) were used in all cases. A Launcher coronary guide catheter (Medtronic, Minneapolis, USA) without side holes was used in all cases. Data acquisition included electrocardiographic signal recording. After intracoronary nitrates $(300 \mu \mathrm{g})$ and acquisition of coronary angiograms, aortic pressure $(\mathrm{Pa})$ and intracoronary distal pressure $(\mathrm{Pd})$ were recorded in the following pattern. First, the pressure wire was zeroed and equalized, and its correct equalization ( $\mathrm{Pd} / \mathrm{Pa}$ ratio of $1.00 \pm 0.01$ ) was confirmed during a 10 -second acquisition in the ascending aorta in each case. Afterward, the pressure sensor was positioned distal to the index stenosis and the guiding catheter was flushed with sa- line and disengaged from the coronary ostium. Baseline pressures were recorded for at least $20 \mathrm{~s}$ before inducing hyperemia. Adenosine administration through a femoral or antecubital fossa vein at a rate of 140 for a minimum of 3 min and a pressure wire pullback maneuver to check for pressure drift were mandatory. Firstly, we performed an infusion through the antecubital vein. After waiting for the wash-out period of adenosine and $\mathrm{Pd} / \mathrm{Pa}$ returned to its baseline value, $300 \mu \mathrm{g}$ of nitrates was given, followed by a saline flush of the guiding catheter. Then, an adenosine infusion through the femoral vein at $140 \mu \mathrm{g} /$ $\mathrm{kg} / \mathrm{min}$ for the same lesion assessment was performed. In the same pressure recording, the bookmarks for core laboratory analyses were placed: 1) when adenosine infusion started; 2) when the pullback maneuver started; and 3) when the pressure sensor reached the tip of the guiding catheter. If a $\mathrm{Pd} / \mathrm{Pa}$ ratio $<0.99$ or $>1.01$ at the catheter tip was documented, the protocol mandated repeat assessment. This stepwise protocol was used for the assessment of every single coronary artery.

The FFR was experimentally and clinically validated under conditions of maximum and stable hyperemia [10] and was automatically calculated by software (ver. 2.4.1.2723, Volcano) as the minimum $\mathrm{Pd} / \mathrm{Pa}$ ratio found in the pressure recording. However, during intravenous adenosine infusion, the minimum hyperemic $\mathrm{Pd} / \mathrm{Pa}$ ratio might develop before stabilization of hyperemia. Hence, conforming to its original validation [11, 12], core laboratory analyses included a thorough review of pressure recordings to corroborate that FFR was calculated: 1) after initiation of adenosine infusion; 2) within stable hyperemia; and 3) before the pullback maneuver. Stable hyperemia was defined as the plateau in mean $\mathrm{Pa}$ after stabilization of changing hemodynamics following the initiation of adenosine infusion and before the pullback maneuver [10]. If a plateau was not clearly observed, stable hyperemia was then defined as the period of pressure recording in which no further systematic fall in Pa was observed, following the initiation of adenosine infusion but before the initiation of the pullback [10]. Within stable hyperemia, the minimum $\mathrm{Pd} / \mathrm{Pa}$ ratio was then labeled as FFR. Core laboratory analyses included an evaluation of pressure waveforms to confirm that none of the following exclusion criteria were present: inappropriate normalization of the pressure wire $(\mathrm{Pd} / \mathrm{Pa}$ ratio < 0.99 or > 1.01), ECG artifacts or significant arrhythmias in the first $20 \mathrm{~s}$ of the recording, loss of Pa or Pd signals at any point during the recording, automatic calculation pitfalls (identification of FFR during ectopic beats, Pa or $\mathrm{Pd}$ noise, wire whipping artifacts, etc.), dampening of $\mathrm{Pa}$ or Pd waveforms, pressure drift higher than $<0.99$ or $>1.01$, and absence of ECG or pressure-pullback recording. The core laboratory also assessed the time to reaction, defined as the time period from the beginning of adenosine infusion to the initial drop of $\mathrm{Pd} / \mathrm{Pa}$, and time to 
Table I. Study population and procedural data $(n=50)$

\begin{tabular}{|c|c|}
\hline Parameter & Value \\
\hline Age [years] & $66.0 \pm 9.3$ \\
\hline Male sex & $36(72.0)$ \\
\hline Height $[\mathrm{cm}]$ & $169.9 \pm 7.9$ \\
\hline Weight [kg] & $80.4 \pm 13.3$ \\
\hline Body mass index $\left[\mathrm{kg} / \mathrm{m}^{2}\right]$ & $27.8 \pm 3.7$ \\
\hline Arterial hypertension & $50(100.0)$ \\
\hline Diabetes mellitus & $28(56.0)$ \\
\hline Previous myocardial infarction & $26(52.0)$ \\
\hline Previous $\mathrm{PCl}$ & $24(48.0)$ \\
\hline Previous CABG & $0(0.0)$ \\
\hline Peripheral arterial disease & $2(4.0)$ \\
\hline Chronic obstructive pulmonary disease & $1(2.0)$ \\
\hline Previous stroke/TIA & $0(0.0)$ \\
\hline Hyperlipidemia & $50(100.0)$ \\
\hline Smoking & $20(40.0)$ \\
\hline Serum creatinine $[\mu \mathrm{mol} / \mathrm{l}]$ & $91.1 \pm 19.4$ \\
\hline LVEF (\%) & $52.8 \pm 8.1$ \\
\hline Heart rate [beat/min] & $71.5 \pm 9.7$ \\
\hline \multicolumn{2}{|l|}{ Angina symptoms - CCS class: } \\
\hline I & $6(12.0)$ \\
\hline$\|$ & $40(80.0)$ \\
\hline III & $4(8.0)$ \\
\hline \multicolumn{2}{|l|}{ Heart failure symptoms - NYHA class: } \\
\hline I & $43(86.0)$ \\
\hline$\|$ & $5(10.0)$ \\
\hline III & $1(4.0)$ \\
\hline IV & $1(4.0)$ \\
\hline \multicolumn{2}{|l|}{ Access: } \\
\hline Radial & $35(70.0)$ \\
\hline Femoral & $15(30.0)$ \\
\hline Number of assessed vessels & $3.0(2.0-4.0)$ \\
\hline \multicolumn{2}{|l|}{ Scheduled treatment: } \\
\hline Conservative & $21(42.0)$ \\
\hline $\mathrm{PCl}$ & $23(46.0)$ \\
\hline CABG & $6(12.0)$ \\
\hline
\end{tabular}

Values presented as number (percentage), mean \pm standard deviation or medi an (interquartile range). CABG - coronary artery bypass grafting, CCS - Canadian Cardiovascular Society, LVEF - left ventricle ejection fraction, NYHA - New York Heart Association, $\mathrm{PCl}$ - percutaneous coronary intervention, TIA - transient ischemic attack. peak hyperemia, defined as the time from the beginning of adenosine infusion to the lowest stable Pd/Pa value.

Quantitative coronary angiography was performed by an independent core laboratory analyzer blinded to the results of FFR. Using the guide catheter for calibration and an edge detection system (CAAS 5.7 QCA system, Pie Medical), the reference vessel diameter and minimum lumen diameter were measured, and the percent diameter stenosis was calculated.

\section{Statistical analysis}

Categorical variables were expressed as numbers of patients (percentages). Continuous variables were expressed as mean \pm standard deviation (SD). Non-normally distributed data were reported as median (interquartile range $(\mathrm{IQR}))$. Agreement among tested methods was assessed by Bland-Altman plots and $95 \%$ limits of agreement. All tests were 2 -tailed, and a $p$-value $<0.05$ was considered statistically significant. All statistical analyses were performed using Statistica 12.0 (StatSoft Inc, Tulsa, OK).

\section{Results}

\section{Study population}

Fifty patients with 125 borderline coronary artery stenoses were enrolled. Baseline patients' and lesion characteristics are presented in Tables I and II. Overall, mean age was $66.0 \pm 9.3$ years, and $72 \%$ of patients were male. All patients presented with stable angina, which was an indication for the coronary angiography. The left anterior descending artery was the most commonly interrogated vessel $(36.8 \%)$.

\section{Procedural data}

Procedural success was $100 \%$ for advancing the pressure wire distally to the stenosis. In general, the study population was composed of coronary stenoses of intermediate angiographic severity (diameter stenosis 44.2 \pm 11.7 by quantitative angiographic assessment). Figure 1 shows the distribution of the FFR values in the study, obtained from femoral infusion. The mean FFR was 0.82 \pm 0.09 . There were no major procedure-related complications. Adenosine caused an asymptomatic transient atrioventricular block of third degree in $5.8 \%$ of patients. Chest pain occurred in $13.6 \%$ of patients. Based on FFR assessment, $42 \%$ of patients were scheduled for conservative treatment, $46 \%$ were treated with $\mathrm{PCl}$ and $12 \%$ were scheduled for coronary artery bypass surgery. In patients who underwent FFR-guided PCI, FFR after the procedure was $0.87 \pm 0.02$ (median 0.87 with IQR 0.86-0.9).

\section{FFR findings and analysis}

The mean FFR for femoral vein adenosine infusion at $140 \mu \mathrm{g} / \mathrm{kg} / \mathrm{min}$ was $0.82 \pm 0.09$ (median: 0.83, IQR: $0.77-0.88$ ) and for antecubital fossa vein adenosine in- 
fusion at $140 \mu \mathrm{g} / \mathrm{kg} / \mathrm{min} 0.82 \pm 0.09$ (median: 0.83, IQR: 0.76-0.89).

The percentage of functionally significant lesions according to different methods of adenosine administration are presented in Figure $2 \mathrm{~A}$. Figure $2 \mathrm{~B}$ shows the scatterplot of the relationship of FFR values for $140 \mu \mathrm{g} /$ $\mathrm{kg} / \mathrm{min}$ femoral, antecubital fossa vein adenosine infusion. There was a strong correlation between FFR values obtained from $140 \mu \mathrm{g} / \mathrm{kg} / \mathrm{min}$ femoral and antecubital fossa vein infusion ( $r=0.99 ; p<0.001$, Figure 3$)$.

The mean time from the initiation of adenosine infusion to the beginning of pressure gradient drop was monitored and was significantly shorter when measured during $140 \mu \mathrm{g} / \mathrm{kg} / \mathrm{min}$ femoral vein infusion as compared to $140 \mu \mathrm{g} / \mathrm{kg} / \mathrm{min}$ antecubital vein infusion (31 $\pm 14 \mathrm{~s}$; median: 28, IQR: 21-37 s vs. $46 \pm 18$ s; median: 44, IQR: 34-50 s; $p<0.001)$.

Also, the time from the initiation of adenosine infusion to the maximal stable hyperemia was significantly shorter for $140 \mu \mathrm{g} / \mathrm{kg} / \mathrm{min}$ femoral vein infusion as compared to $140 \mu \mathrm{g} / \mathrm{kg} / \mathrm{min}$ antecubital vein infusion (49 \pm 19 s; median: 46, IQR: $35-58$ s vs. $68 \pm 23$ s; median: 63, IQR: 53-76 s; $p<0.001)$ - Figure 4. Additionally, we performed a paired differences analysis comparing $140 \mu \mathrm{g} / \mathrm{kg} / \mathrm{min}$ femoral with $140 \mu \mathrm{g} / \mathrm{kg} / \mathrm{min}$ antecubital fossa vein infusion and found no difference between femoral and antecubital infusion FFR values with mean delta of 0.001 ( $95 \% \mathrm{Cl}:-0.003$ to $0.0005 ; p=0.16)$.

\section{Discussion}

Our study identified the optimal way and dose of adenosine administration for the reliable measurement of coronary FFR for assessment of the hemodynamic severity of borderline coronary stenoses. Femoral and antecubital fossa vein infusion showed almost the same but not

A

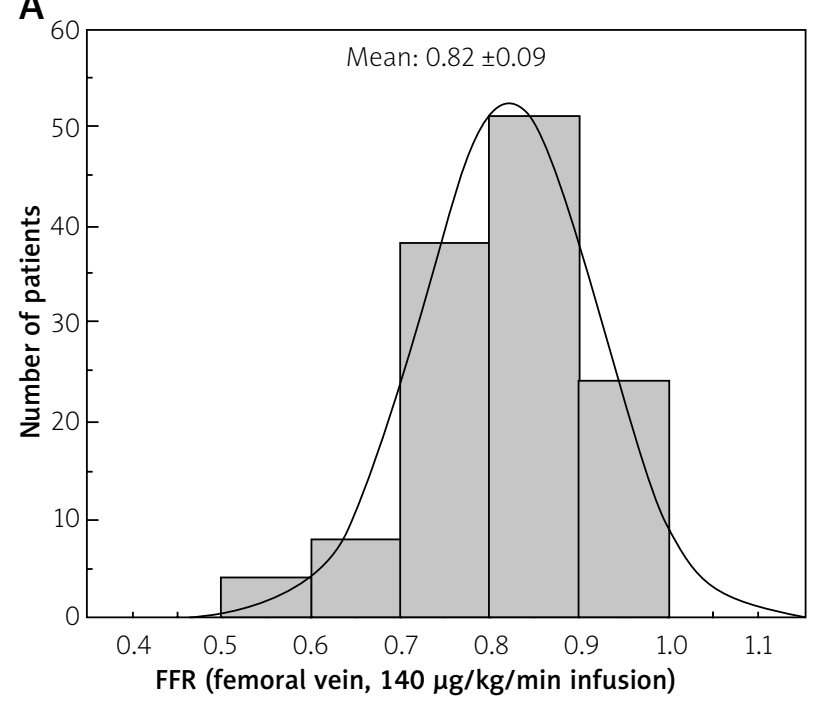

Table II. Lesion characteristics $(n=125)$

\begin{tabular}{|c|c|}
\hline Lesions & Value \\
\hline \multicolumn{2}{|l|}{ Vessel: } \\
\hline LAD & $48(38.4)$ \\
\hline$\overline{D g}$ & $11(8.8)$ \\
\hline$C x$ & $32(25.6)$ \\
\hline $\mathrm{Mg}$ & $9(7.2)$ \\
\hline RCA & $25(20.0)$ \\
\hline \multicolumn{2}{|c|}{ Quantitative coronary angiography results: } \\
\hline Lesion length [mm] & $21.7 \pm 14.0$ \\
\hline $\mathrm{RVD}[\mathrm{mm}]$ & $2.6 \pm 0.6$ \\
\hline$\overline{M L D}[\mathrm{~mm}]$ & $1.4 \pm 0.4$ \\
\hline DS (\%) & $44.2 \pm 11.7$ \\
\hline Eccentric lesion & $67(53.6)$ \\
\hline Moderate/severe tortuosity & $52(41.6)$ \\
\hline Irregular contours & $11(9.2)$ \\
\hline Moderate/severe calcifications & $49(40.8)$ \\
\hline Ostial lesion & $11(9.2)$ \\
\hline
\end{tabular}

Values presented as number (percentage) or mean \pm standard deviation $C x$ - circumflex artery, Dg-diagonal branch, DS - diameter stenosis, LAD - left anterior descending artery, LMCA - left main coronary artery, Mg - marginal branch, $M L D$ - minimal lumen diameter, $R C A$ - right coronary artery, RVD - reference vessel diameter.

identical FFR values after achieving maximal and stable hyperemia. However, no systematic direction of bias was evident from Bland-Altman analysis. Moreover, maximal hyperemia takes longer to obtain with the antecubital vein compared to a large peripheral vein.

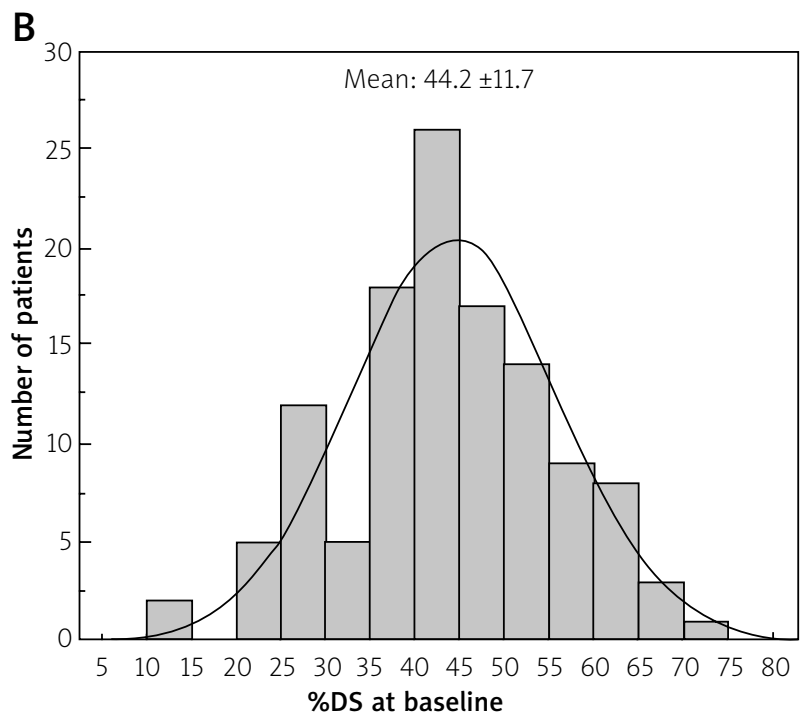

Figure 1. Distribution of the \%DS (A) and fractional flow reserve (B) values in the study population $\% D S$ - percent diameter stenosis, FFR - fractional flow reserve. 
A

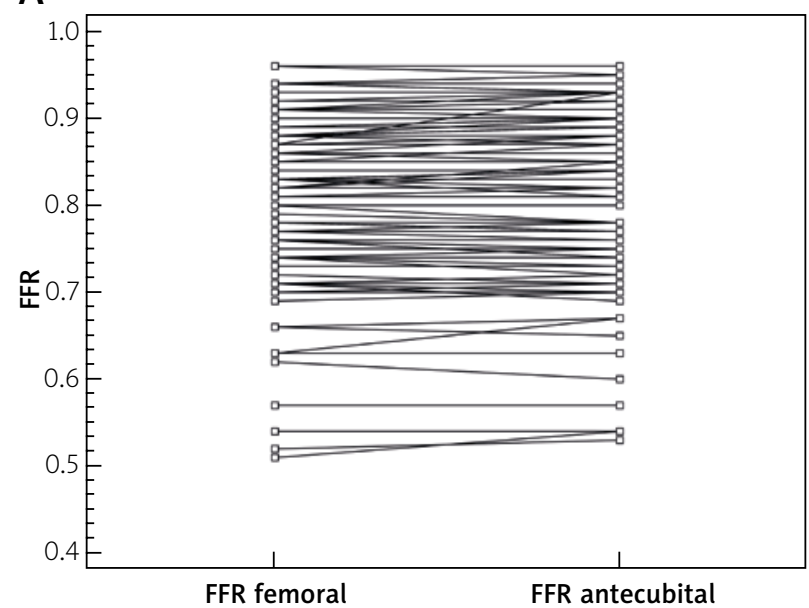

B

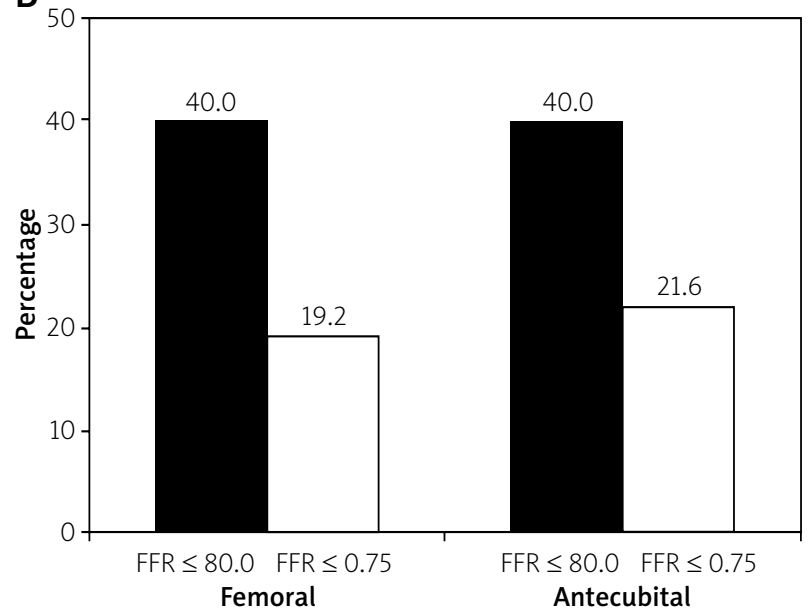

Figure 2. A - Scatterplot presenting fractional flow reserve (FFR) values for $140 \mu \mathrm{g} / \mathrm{kg} / \mathrm{min}$ femoral and antecubital fossa vein adenosine infusion. B - Percentage of functionally significant lesions according to different methods of adenosine administration

A

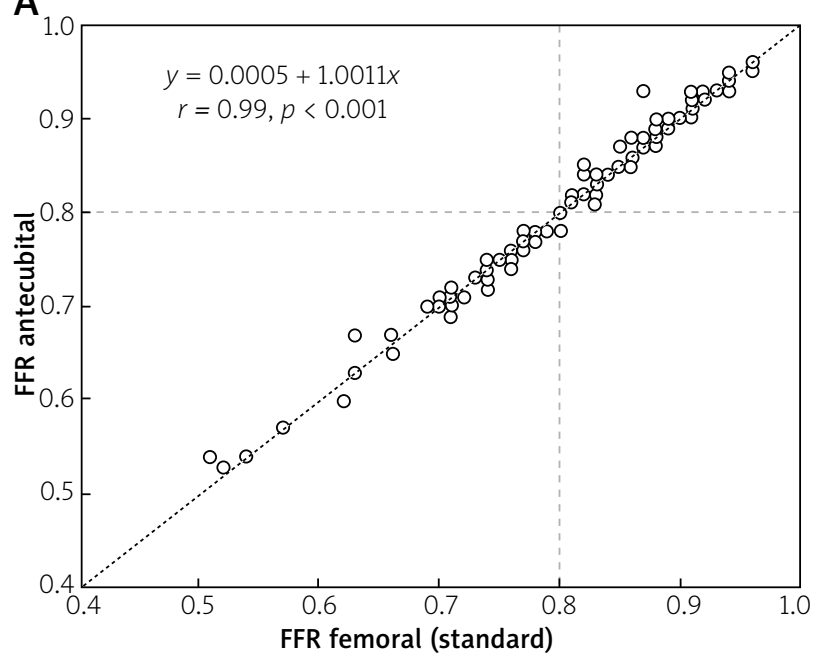

B Bias $0.001(95 \% \mathrm{Cl}$ limits of aggrement from -0.021 to 0.024$)$

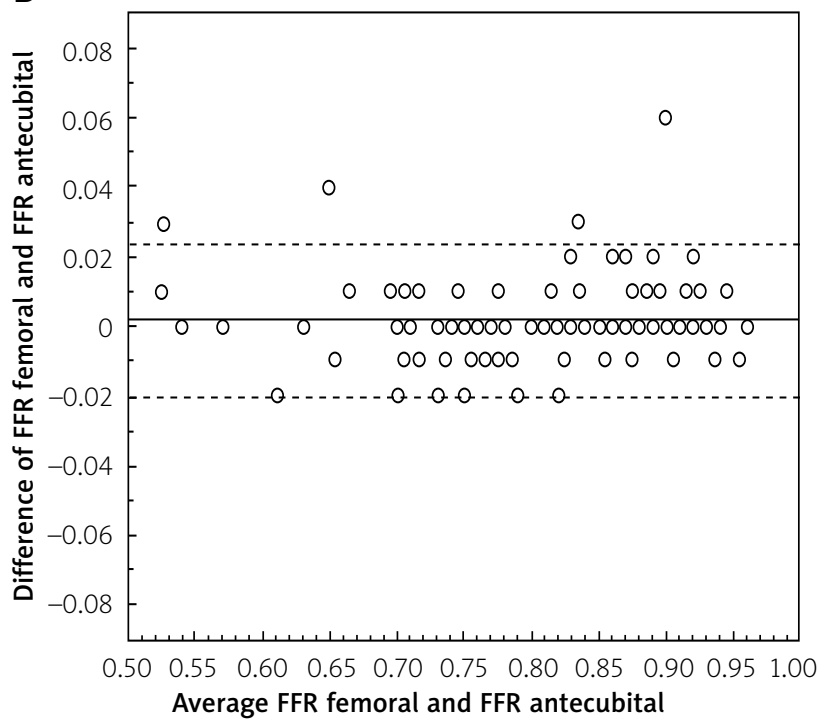

Figure 3. A - Correlation of fractional flow reserve (FFR) values between $140 \mu \mathrm{g} / \mathrm{kg} / \mathrm{min}$ femoral and $140 \mathrm{\mu g} / \mathrm{kg} / \mathrm{min}$ antecubital fossa vein adenosine infusion. B - Bland-Altman plot for the same data

This finding, in our opinion, is crucial and timely, as in everyday clinical practice there still seem to be questions and doubts about the optimal way of intravenous adenosine administration.

Coronary pressure-derived FFR is the current standard of care for the functional assessment of lesion severity in patients with intermediate-grade stenosis (typically around $40-90 \%$ stenosis) without evidence of ischemia in non-invasive testing, or in those with multivessel disease [13].

The value 0.80 is the accepted FFR cut-off for defining hemodynamically relevant lesions requiring revascularization. However, the FFR threshold of 0.75 is also used to define more severe ischemia that is of prognostic rel- evance $[5,6]$. In contrast to previously published data, in our study, we assessed more "severe" moderate lesions with FFR values $<0.80$ and $<0.75[8,9]$ and confirmed the utility of peripheral vein adenosine infusion.

Central (femoral) venous infusion of adenosine has been the gold standard method of obtaining hyperemia for FFR measurement [7, 14-17] and could induce hyperemia with more reliable hyperemic efficacy than intracoronary bolus injection [18-20]. However, it requires an additional puncture of the femoral vein, which may increase the risk of vascular complications. Also, it is not so convenient to use during the transradial approach, which is the method of choice in the majority of catheterization laboratories worldwide, especially in patients 
A

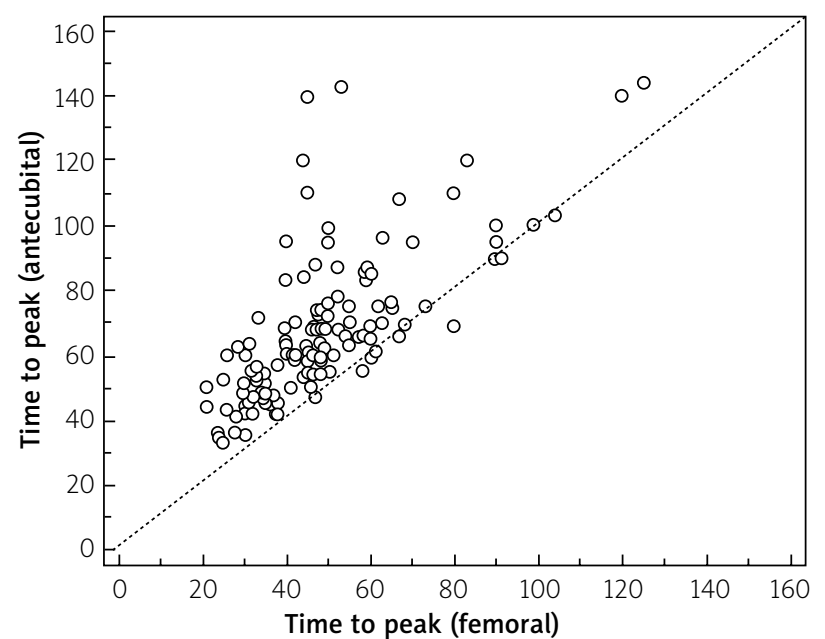

B

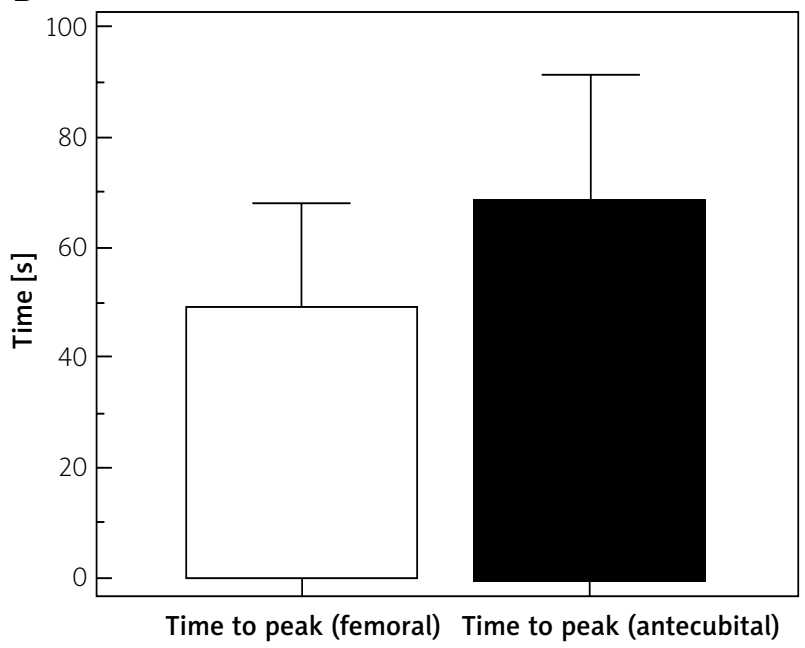

C

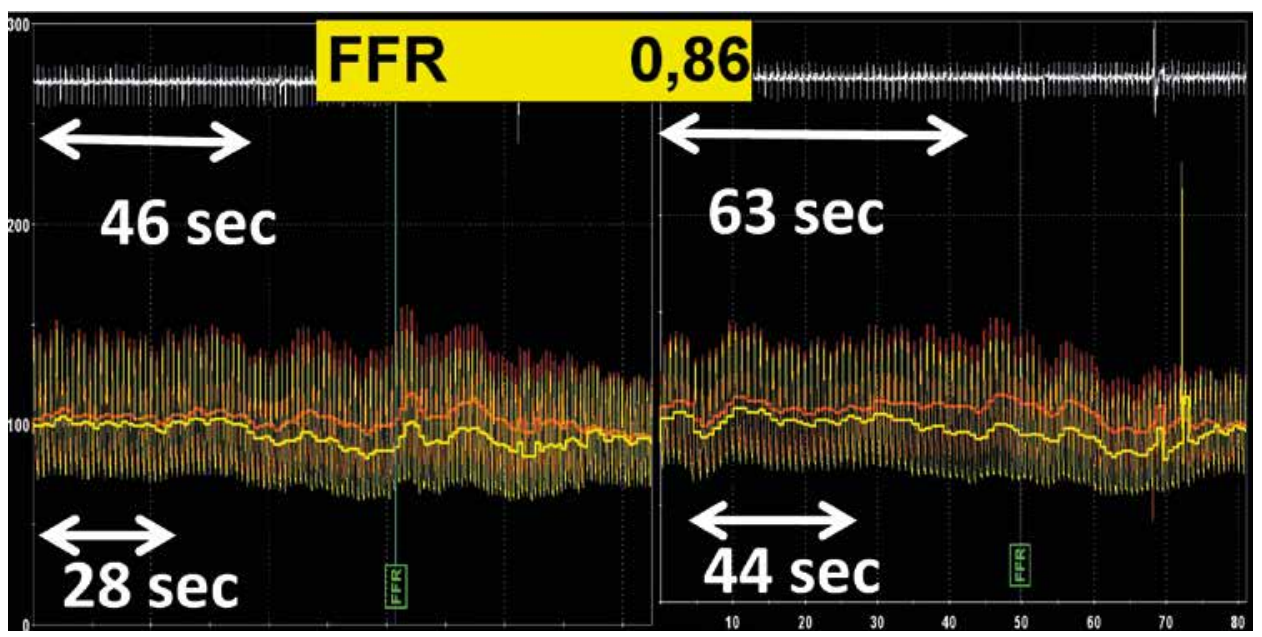

Figure 4. A - Femoral and antecubital fossa vein adenosine administration for individual cases with the line of identity. B - Mean times to peak hyperemia with femoral and antecubital fossa vein adenosine infusion. C - Exemplary case of fractional flow reserve (FFR) assessment with femoral and antecubital fossa vein adenosine infusion

scheduled for elective coronary angiography. The use of a combination of the transradial access and FFR-based lesion assessment is rapidly becoming a standard of care in many clinical situations, and the issue of adenosine delivery and the dose is therefore relevant and timely. Therefore an increasing frequency of peripheral venous adenosine administration for FFR has been noted, despite doubts whether a small cannula would actually generate adequate and sufficiently stable coronary hyperemia compared with a central venous infusion. In our study, we selected the antecubital fossa vein as a route of adenosine infusion, as this site is the most common in patients undergoing coronary angiography. To provide adequate hyperemia, we used a 20-gauge needle in each case. In our study, antecubital vein infusion of adenosine was as effective as femoral vein infusion in the induction of maximal hyperemia. There was no difference in mean FFR between these two ways of adenosine administration, and the number of functionally significant stenoses was not different between femoral vein and antecubital vein infusions of adenosine. In addition to FFR, the mean hyperemic transit time was also compared, and we found that the time to maximal hyperemia was longer with antecubital vein infusion of adenosine than with femoral vein infusion. In our study, the mean difference of time to maximal hyperemia was $14 \mathrm{~s}$, comparable to data previously published $[8,9]$. Our results together with previously available data suggest that the infusion time should be long enough (at least $2 \mathrm{~min}$ ) when the antecubital vein is selected for adenosine infusion for FFR assessment. Our results are in agreement with previous studies; however, we have assessed more lesions [8, 9]. Our findings support a strategy of using peripheral venous adenosine administration as the default approach. The occasional FFR difference between access routes may actually be due to the intrinsic variability of the test itself and independent of whether adenosine is administered via peripheral and central vein infusion [21]. 


\section{Study limitations}

The FFR procedures were performed by two experienced operators at a single center but interobserver variability was not assessed. However, individual operator technique was formalized using local and study guidelines, and data analysis was performed by a core laboratory blinded to the results. We did not compare infusion of adenosine from differing peripheral access sites (e.g., back of the hand, wrist, and forearm). Ostial lesions of the right coronary artery or left main as well as tandem lesions were not included. In this study, we also did not compare results with intracoronary adenosine administration.

\section{Conclusions}

Antecubital fossa vein adenosine infusion achieved FFR values are very similar to those obtained using femoral vein adenosine administration. However, time to maximal hyperemia is longer with infusion via the antecubital vein.

\section{Acknowledgments}

The study was supported by the grant from the Jagiellonian University Medical College (K/ZDS/005469).

\section{Conflict of interest}

The authors declare no conflict of interest.

\section{References}

1. Bech GJ, De Bruyne B, Pijls NH, et al. Fractional flow reserve to determine the appropriateness of angioplasty in moderate coronary stenosis: a randomized trial. Circulation 2001; 103: 2928-34.

2. De Bruyne B, Pijls NH, Kalesan B, et al. Fractional flow reserveguided $\mathrm{PCl}$ versus medical therapy in stable coronary disease. N Engl J Med 2012; 367: 991-1001.

3. Pijls NH, van Schaardenburgh P, Manoharan G, et al. Percutaneous coronary intervention of functionally non-significant stenosis: 5-year follow-up of the DEFER Study. J Am Coll Cardiol 2007; 49: 2105-11.

4. Tonino PA, De Bruyne B, Pijls NH, et al. Fractional flow reserve versus angiography for guiding percutaneous coronary intervention. N Engl J Med 2009; 360: 213-24.

5. Neumann FJ, Sousa-Uva M, Ahlsson A, et al. 2018 ESC/EACTS Guidelines on myocardial revascularization. Kardiol Pol 2018; 76: 1585-664.

6. Johnson NP, Toth GG, Lai D, et al. Prognostic value of fractional flow reserve: linking physiologic severity to clinical outcomes. J Am Coll Cardiol 2014; 64: 1641-54.

7. Pijls NH, Fearon WF, Tonino PA, et al. Fractional flow reserve versus angiography for guiding percutaneous coronary intervention in patients with multivessel coronary artery disease: 2-year follow-up of the FAME (Fractional Flow Reserve Versus Angiography for Multivessel Evaluation) study. J Am Coll Cardiol 2010; 56: 177-84.

8. Barbero U, D’Ascenzo F, Campo G, et al. Safety of FFR-guided revascularisation deferral in Anatomically prognostiC diseasE
(FACE: CARDIOGROUP V STUDY): a prospective multicentre study. Int J Cardiol 2018; 270: 107-12.

9. Kern MJ, Deligonul U, Tatineni S, et al. Intravenous adenosine: continuous infusion and low dose bolus administration for determination of coronary vasodilator reserve in patients with and without coronary artery disease. J Am Coll Cardiol 1991; 18: 718-29.

10. Scott P, Sirker A, Dworakowski R, et al. Fractional flow reserve in the transradial era: will hand vein adenosine infusion suffice? A comparative study of the extent, rapidity, and stability of hyperemia from hand and femoral venous routes of adenosine administration. JACC Cardiovasc Interv 2015; 8: 527-35.

11. Seo MK, Koo BK, Kim JH, et al. Comparison of hyperemic efficacy between central and peripheral venous adenosine infusion for fractional flow reserve measurement. Circ Cardiovasc Interv 2012; 5: 401-5.

12. Pijls NH, van Son JA, Kirkeeide RL, et al. Experimental basis of determining maximum coronary, myocardial, and collateral blood flow by pressure measurements for assessing functional stenosis severity before and after percutaneous transluminal coronary angioplasty. Circulation 1993; 87: 1354-67.

13. Echavarria-Pinto $M$, Petraco R, van de Hoef $T$, et al. Fractional flow reserve and minimum $\mathrm{Pd} / \mathrm{Pa}$ ratio during intravenous adenosine infusion: very similar but not always the same. Eurolntervention 2016; 11: 1013-9.

14. de Bruyne B, Bartunek J, Sys SU, et al. Simultaneous coronary pressure and flow velocity measurements in humans. Feasibility, reproducibility, and hemodynamic dependence of coronary flow velocity reserve, hyperemic flow versus pressure slope index, and fractional flow reserve. Circulation 1996; 94: 1842-9.

15. Pijls NH, de Bruyne B, Peels K, et al. Measurement of fractional flow reserve to assess the functional severity of coronary-artery stenoses. N Engl J Med 1996; 334: 1703-8.

16. Pijls NH, Van Gelder B, Van der Voort P, et al. Fractional flow reserve: a useful index to evaluate the influence of an epicardial coronary stenosis on myocardial blood flow. Circulation 1995; 92: 3183-93.

17. De Bruyne B, Pijls NH, Barbato E, et al. Intracoronary and intravenous adenosine 5 -triphosphate, adenosine, papaverine, and contrast medium to assess fractional flow reserve in humans. Circulation 2003; 107: 1877-83.

18. Jeremias A, Whitbourn R, Filardo S, et al. Adequacy of intracoronary versus intravenous adenosine-induced maximal coronary hyperemia for fractional flow reserve measurements. Am Heart J 2000; 140: 651-7.

19. Jeremias A, Filardo S, Whitbourn R, et al. Effects of intravenous and intracoronary adenosine 5 -triphosphate as compared with adenosine on coronary flow and pressure dynamics. Circulation 2000; 101: 318-23.

20. Lopez-Palop R, Saura D, Pinar E, et al. Adequate intracoronary adenosine doses to achieve maximum hyperaemia in coronary functional studies by pressure derived fractional flow reserve: a dose response study. Heart 2004; 90: 95-6.

21. Petraco R, Sen S, Nijjer S, et al. Fractional flow reserve-guided revascularization: practical implications of a diagnostic gray zone and measurement variability on clinical decisions. JACC Cardiovasc Interv 2013; 6: 222-5. 